\title{
Aumentando a Eficiência na Execução de Algoritmos de Grafos em HPC
}

\author{
Marcelo K. Moori ${ }^{1}$, Hiago Mayk G. de A. Rocha ${ }^{1}$, Janaina Schwarzrock ${ }^{1}$, \\ Arthur F. Lorenzon ${ }^{2}$ e Antonio Carlos S. Beck ${ }^{1}$ \\ ${ }^{1}$ Universidade Federal do Rio Grande do Sul - Porto Alegre - RS - Brasil \\ ${ }^{2}$ Universidade Federal do Pampa - Alegrete - RS - Brasil \\ mkmooridinf.ufrgs.br
}

\begin{abstract}
The growing need for extracting information from massive data structured as graphs - has been pushing the development of even more robust parallel algorithms for such processing. However, the algorithms' communicationbound behavior and the highly irregular structure of the currently used graphs are hurdles to achieving the same levels of performance and efficiency as observed for other parallel applications. In this paper, we show that the scalability of different graph applications varies according to the used algorithms and their databases and, in most cases, using all available cores (i.e., all available processor's cores for execution) is not the best option in terms of efficiency. Based on that, we propose MultGraph, a framework that enables the simultaneous processing of several algorithms/graphs, distributing them in a non-uniform way among the cores, instead of executing them serially (i.e., one after another) with the maximum available parallelism. MultGraph works in two steps: (i) characterizing the algorithms/graphs by their efficiency levels; (ii) defining the allocations (clusters of algorithms to be executed concurrently), number of threads for each of them, and clusters' execution order. Experimental results on three multicore processors (Intel and AMD) show that MultGraph improves in up to $9.21 \times$ and on average $4.52 \times$ the algorithm's execution time compared to the default execution of applications in HPC systems.
\end{abstract}

Resumo. A crescente necessidade de extrair informações de dados massivos - estruturados como grafos - tem impulsionado o desenvolvimento de algoritmos paralelos cada vez mais robustos para este processamento. No entanto, o comportamento voltado à comunicação e a estrutura altamente irregular dos grafos usados cotidianamente são obstáculos para alcançar os mesmos níveis de desempenho e eficiência como os observados em outras aplicações paralelas. Neste artigo, nós mostramos que a escalabilidade de diferentes aplicações de grafos variam de acordo com o algoritmo usado e a sua base de dados e que, em muitos casos, utilizar todos recursos disponíveis (i.e. todos os núcleos do processador para a execução) não é a melhor opção em termos de eficiência. Com base nisso, nós propomos o MultGraph, um framework que permite o processamento simultâneo de vários algoritmos/grafos, distribuindo-os de maneira não uniforme entre os núcleos, ao invés de executá-los serialmente (i.e. um após o outro) com o máximo paralelismo disponível. MultGraph funciona em dois passos: (i) caracterizando os algoritmos/grafos pelos seus níveis de eficiência; (ii) definindo as alocações (agrupamentos de algoritmos e entradas a serem executados concorrentemente), número de threads para cada um deles, e a ordem de execução destes grupos. Resultados experimentais em três processadores multicore (Intel e AMD) mostram que o MultGraph melhora em até $9,21 \times e$ 4, $52 \times$ 
em média o tempo de execução das aplicações em relação à execução padrão de aplicações em sistemas HPC.

\section{Introdução}

O aumento no número de núcleos e memória disponível nos Computadores de Alto Desempenho (do inglês, High Performance Computing - HPC) vem possibilitando a análise de uma quantidade massiva de dados estruturados em forma de grafos, extraídos de fontes como Google, Facebook e Twitter [Yan et al. 2017, Mofrad et al. 2020]. Assim, por meio de algoritmos como o PageRank e Caminhos Mínimos, é possível realizar operações como o ordenamento das páginas mais visitadas em uma busca de conteúdo no Google ou até a realização de computações mais complexas no âmbito da Inteligência Artificial. Enquanto a necessidade de processar os grafos do mundo real de forma eficiente impulsionou o desenvolvimento de vários frameworks, como Ligra e Polymer [Shun and Blelloch 2013, Zhang et al. 2015], as características intrínsecas dos sistemas de HPC (e.g., hierarquia de memória complexa) e dos grafos (e.g., estrutura irregular e dimensão) têm desafiado os programadores a extrair todo o potencial dos recursos de processamento disponíveis [Rocha et al. 2021].

Tradicionalmente, a maneira de executar aplicações paralelas (que inclui processamento de grafos) nos sistemas de HPC é submeter jobs que executem a aplicação com o número de threads igual ao máximo número de recursos de processamento disponíveis no sistema. Porém, nem todas as aplicações se beneficiam disso, o que significa que usar o máximo número de threads pode não ser eficiente ${ }^{1}$ [Lorenzon and Beck Filho 2019], isto é, não utilizar os recursos computacionais disponíveis de maneira ótima. Essa falta de escalabilidade está relacionada tanto a fatores de hardware (e.g, saturação de unidades funcionais e barramento de comunicação) quanto de software (e.g, sincronização de dados e acessos concorrentes à memória compartilhada) [Raasch and Reinhardt 2003, Suleman et al. 2008, da Silva et al. 2020]. Além do mais, aplicações de grafos são intrinsecamente orientadas à comunicação: possuem uma baixa fração de computação em relação às comunicações realizadas entre as threads [Mofrad et al. 2020], que envolve acessos extras à memória e/ou troca de mensagens [Beamer et al. 2015b]. Essa característica intensifica os problemas acima mencionados, uma vez que as unidades de processamento tendem a ficar ociosas por causa das sincronizações excessivas.

Para ilustrar melhor a discussão acima, a Tabela 1 mostra o número de threads (\#Thr) que apresenta o melhor tempo de execução seguindo da eficiência do sistema (Eff) quando os algoritmos Betweenness Centrality (BC) e Breadth-First Search (BFS) executam diferentes grafos (discutidos em detalhes na seção 2). Pode-se observar que o melhor número de threads para executar cada aplicação varia de acordo com o grafo processado; e que o mesmo grafo pode influenciar na escalabilidade das aplicações, e.g., o BFS executando Texas é escalável até 18 threads, enquanto que o BC com Texas escala até 30 threads. Outra limitação que destacamos é com relação a eficiência do sistema: o comportamento ideal (desejado) para uma aplicação que escala é apresentar uma suave redução na eficiência do sistema conforme aumenta o número de threads [Eager et al. 1989], como é o caso da BC-orkut com 35\%, fazendo uso do máximo de threads. Contudo, na maioria dos casos, acontece uma redução brusca na eficiência.

Uma vez que nem todas as aplicações de grafos se beneficiam ao usar o máximo de recursos do sistema, como visto nos cenários anteriores, ajustar adequadamente o número de threads resulta no aumento de eficiência dessas aplicações. Assim, ao mesmo tempo

\footnotetext{
${ }^{1}$ Um sistema é $100 \%$ eficiente quando o seu ganho em desempenho é equivalente ao número de núcleos usados para executar sua versão paralelizada (e.g., usando-se 16 núcleos obtém-se 16x de speedup), mas ele apresenta uma eficiência de $50 \%$ se ao usar 16 cores obtém-se um speedup de 8x.
} 
Tabela 1. Variação do melhor número de threads e eficiência do sistema nas execuções dos algoritmos BC e BFS conforme a variação dos grafos de entrada. Experimentos executados no Intel Xeon E5-2640v2.

\begin{tabular}{|c|c|c|c|c|c|c|c|c|c|c|}
\hline & Amazon & California & Google & Pennsyl. & Youtube & Berk & Texas & Wikitalk & Orkut & Patent \\
\hline BC (\#Thr / Eff.) & $10 / 21 \%$ & $30 / 16 \%$ & $32 / 26 \%$ & $26 / 14 \%$ & $28 / 11 \%$ & $18 / 16 \%$ & $30 / 11 \%$ & $30 / 22 \%$ & $32 / 35 \%$ & $14 / 30 \%$ \\
\hline BFS (\#Thr / Eff.) & $6 / 43 \%$ & $30 / 13 \%$ & $30 / 20 \%$ & $18 / 18 \%$ & $16 / 18 \%$ & $14 / 20 \%$ & $18 / 17 \%$ & $24 / 20 \%$ & $30 / 23 \%$ & $14 / 21 \%$ \\
\hline
\end{tabular}

em que uma aplicação está sendo executada com um pequeno número de threads, porém com uma eficiência maior, pode-se utilizar os recursos que estão ociosos para executar outra aplicação. Desta maneira, este artigo baseia-se no princípio que executar simultaneamente múltiplos algoritmos de grafos de modo que a eficiência total do sistema aumente e, portanto, o tempo de execução total de todos os grafos a serem executados diminua. Para isto, neste trabalho propomos o framework MultGraph: uma estratégia de multiprogramação que identifica, dado o conjunto de algoritmos/grafos e o multiprocessador alvo, a melhor combinação de aplicações que deve ser executada simultaneamente. $\mathrm{O}$ algoritmo usado no framework trabalha em dois passos, dado um conjunto de grafos a serem executados: (i) Caracteriza as aplicações de acordo com o número de threads que apresenta melhor eficiência; e (ii) Define a alocação e a ordem de execução das aplicações nos núcleos do sistema.

Em resumo, as principais contribuições deste trabalho são:

- Análise de escalabilidade dos algoritmos de grafos, mostrando que dependendo do tipo de computação e da topologia do grafo, tem-se diferentes número de threads que entregam o melhor tempo de execução e eficiência do sistema;

- O framework MultGraph: uma ferramenta para execução simultânea de aplicações de grafos que oferece ganhos significativos tanto no tempo de execução quanto na eficiência do sistema;

- Resultados experimentais considerando três multiprocessadores diferentes, mostraram que o algoritmo MultGraph consegue melhorar em até $9.21 \times$ e em média $4,52 \times$ o tempo de execução, quando comparada com a execução padrão em sistemas HPC.

O restante deste artigo está organizado como segue: A seção 2 apresenta os conceitos básicos e as características dos algoritmos e grafos avaliados neste trabalho; Os detalhes do algoritmo MultGraph são apresentados na seção 3; A metodologia utilizada é detalhada na seção 4; A seção 5 apresenta os resultados; Os trabalhos relacionados são apresentados na seção 6. Por fim, a seção 7 conclui este trabalho.

\section{Background}

\subsection{Grafos}

Grafos são formados por uma quantidade massiva de dados e variam bastante em suas topologias. O modo como os grafos são lidos e processados está intrinsecamente ligado à distribuição dos seus nodos e arestas em sua estrutura; assim, podemos caracterizá-los com base nas seguintes métricas extraídas do Stanford Network Analysis Platform (SNAP) [Leskovec and Krevl 2014]: Weakly Connected Components (WCC): é um subconjunto maximal dos vértices do grafo com a restrição de que para quaisquer pares de vértices deve existir um caminho entre os vértices do par, ignorando a sua direção; Strongly Connected Components (SCC): é um subconjunto maximal dos vértices do grafo tal qual o WCC, mas com sua direção importando nesse caso (e.g., para um grafo não direcionado o SCC e WCC são equivalentes); Coeficiente Médio de Clusterização (CMC): é a média dos coeficientes de agrupamentos locais de cada vértice do grafo, o que é definido pela proporção de ligações entre os vértices da sua vizinhança e o número de ligações 
Tabela 2. Características dos grafos.

\begin{tabular}{lrrrrrrrrrr}
\hline & Amazon & California & Google & Pennsyl. & Youtube & Berk & Texas & Wikitalk & Orkut & Patent \\
\hline \#Vértices & $3,3 \mathrm{e} 5$ & $1,9 \mathrm{e} 6$ & $8,7 \mathrm{e} 5$ & $1,0 \mathrm{e} 6$ & $1,1 \mathrm{e} 6$ & $6,8 \mathrm{e} 5$ & $1,3 \mathrm{e} 6$ & $2,3 \mathrm{e} 6$ & $3,0 \mathrm{e} 6$ & $3,7 \mathrm{e} 6$ \\
\#Arestas & $9,2 \mathrm{e} 5$ & $2,7 \mathrm{e} 6$ & $5,3 \mathrm{e} 6$ & $1,5 \mathrm{e} 6$ & $2,9 \mathrm{e} 6$ & $7,6 \mathrm{e} 6$ & $1,9 \mathrm{e} 6$ & $5,0 \mathrm{e} 6$ & $1,1 \mathrm{e} 8$ & $1,6 \mathrm{e} 7$ \\
\#Vért. no maior WCC & 1 & 0,996 & 0,977 & 1 & 1 & 0,956 & 0,979 & 0,998 & 1 & 0,997 \\
\#Arres. no maior WCC & 1 & 0,998 & 0,993 & 1 & 1 & 0,987 & 0,978 & 0,999 & 1 & 1 \\
\#Vért. no maior SCC & 1 & 0,996 & 0,497 & 1 & 1 & 0,489 & 0,979 & 0,047 & 1 & 0 \\
\#Ares. no maior SCC & 1 & 0,998 & 0,67 & 1 & 1 & 0,595 & 0,978 & 0,297 & 1 & 0 \\
CMC & $3,9 \mathrm{e}-1$ & $4,6 \mathrm{e}-2$ & $5,1 \mathrm{e}-1$ & $4,6 \mathrm{e}-2$ & $8,0 \mathrm{e}-2$ & $5,9 \mathrm{e}-1$ & $4,7 \mathrm{e}-2$ & $5,2 \mathrm{e}-2$ & $1,6 \mathrm{e}-1$ & $7,5 \mathrm{e}-2$ \\
NT & $6,6 \mathrm{e} 5$ & $1,2 \mathrm{e} 5$ & $1,3 \mathrm{e} 7$ & $6,7 \mathrm{e} 4$ & $3,0 \mathrm{e} 6$ & $6,4 \mathrm{e} 7$ & $8,2 \mathrm{e} 4$ & $9,2 \mathrm{e} 6$ & $6,2 \mathrm{e} 8$ & $7,5 \mathrm{e} 6$ \\
FTF & $7,9 \mathrm{e}-2$ & $2,0 \mathrm{e}-2$ & $1,9 \mathrm{e}-2$ & $2,0 \mathrm{e}-2$ & $2,0 \mathrm{e}-3$ & $2,7 \mathrm{e}-3$ & $2,0 \mathrm{e}-2$ & $1,1 \mathrm{e}-3$ & $1,4 \mathrm{e}-2$ & $2,0 \mathrm{e}-2$ \\
Diâmetro & 44 & 849 & 21 & 786 & 20 & 514 & 1054 & 9 & 9 & 22 \\
90-PDE & 15 & 500 & 8,1 & 530 & 6,5 & 9,9 & 670 & 4 & 4,8 & 9,4 \\
\hline
\end{tabular}

Tabela 3. Características dos algoritmos.

\begin{tabular}{lcccc}
\hline & $\begin{array}{c}\text { Fonte Única } \\
\text { /Todo Grafo }\end{array}$ & (Não-)Ponderado & $\begin{array}{l}\text { \#Vértices } \\
\text { por Iteração }\end{array}$ & $\begin{array}{l}\text { Percorrimento } \\
\text { /Computação }\end{array}$ \\
\hline BC & Fonte Única & Não-Ponderado & Parte & Computação \\
BFS & Fonte Única & Não-Ponderado & Parte & Percorrimento \\
CC & Todo Grafo & Não-Ponderado & Parte & Percorrimento \\
PR & Todo Grafo & Não-Ponderado & Todo & Computação \\
SSSP & Fonte Única & Ponderado & Parte & Percorrimento \\
TC & Todo Grafo & Não-Ponderado & Parte & Percorrimento \\
\hline
\end{tabular}

que poderiam existir entre estes; Número de Triângulos (NT): é o número de triplas (combinação de três vértices) conectadas, ignorando a sua direção; Fração de Triângulos Fechados $(\boldsymbol{F T F})$ : é a razão entre o número de triângulos e o total de triplas (todas as combinações de três vértices conectadas ou não); Diâmetro: o mais longo menor caminho entre qualquer par de vértices do grafo; 90-Percentil de Diâmetro Efetivo (90-PDE): é a menor distância para qual $90 \%$ dos pares de uma amostra de 1000 vértices estão distanciados no máximo por esse valor. Na Tabela 2 nós destacamos essas características nos diferentes grafos avaliados neste trabalho (extraídos do SNAP).

\subsection{Frameworks e Algoritmos para Processamento de Grafos}

Para a extração eficiente de informações em grafos (e.g., conectividade, caminhos mínimos e visibilidade dos vértices), vários trabalhos da literatura propõem frameworks para otimizar a execução paralela de algoritmos de processamento de grafos [Shun and Blelloch 2013, Mofrad et al. 2020]. Tais frameworks apresentam implementações de alto desempenho de algoritmos amplamente utilizados pela indústria, como por exemplo o PageRank (PR) e o Breadth-First Search (BFS), com aplicabilidade em diversas áreas (e.g., química, medicina, comercio e logística) [Gleich 2014].

Os algoritmos amplamente estudados na literatura de grafos e que a maioria dos frameworks implementam são [Heidari et al. 2018]: Betweenness Centrality (BC) aproxima a medida de intermediação do grafo por um subconjuntos de vértices, tendo que a intermediação de um vértice $v$ é dada pela razão entre os caminhos mínimos que atravessam $v$ e todos os caminhamentos mínimos do conjunto considerado; Breadth-First Search (BFS) realiza a travessia do grafo a partir de um vértice raiz, visitando todos os vértices de mesmo nível antes de avançar para o próximo; Connected Components $(\boldsymbol{C C})$ computa todos os componentes conexos do grafo, que é definido por um conjunto de vértices no qual existe um caminho não direcionado entre qualquer par de vértices do conjunto; PageRank (PR) iterativamente calcula o valor de PageRank para todos os vértices com um fator de amortização $d$ de 0,85; Single-Source Shortest Paths (SSSP) calcula todos os caminhamentos mínimos alcançáveis a partir de um dado vértice raiz; Triangle Counting (TC) computa o total de triângulos do grafo.

Além das diferenças topológicas dos grafos do mundo real (ver Tabela 2), os al- 


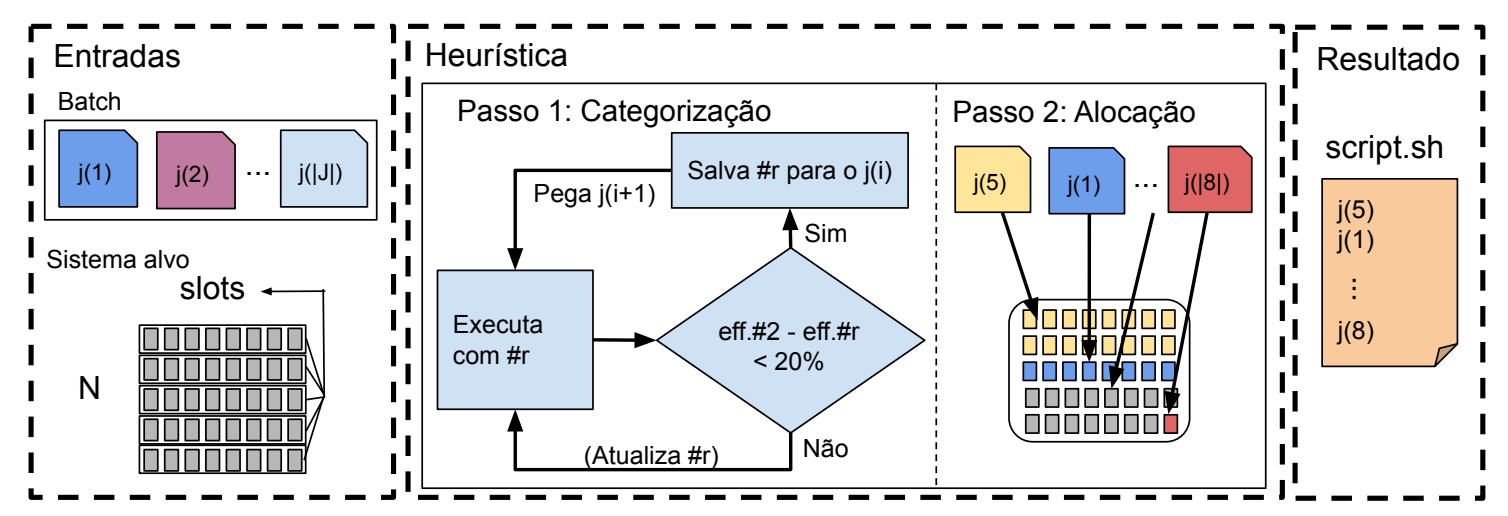

Figura 1. Fluxo de otimização do MultGraph.

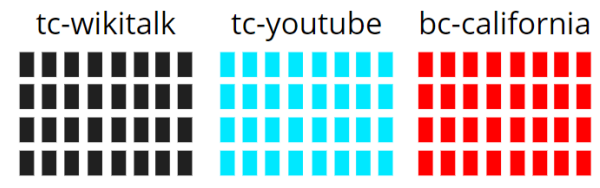

(1)

tempo

(a) Execução Tradicional

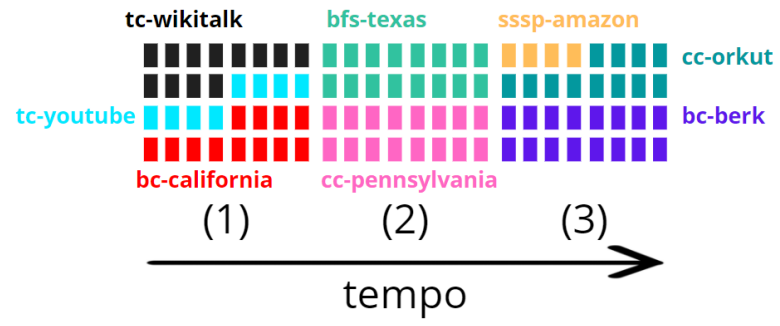

(b) MultGraph

Figura 2. Exemplo de comparação entre a (a) execução tradicional de aplicações de HPC e (b) a solução gerada pelo MultGraph.

goritmos também possuem diferenças em termos de (ver Tabela 3): requerer ou não um vértice inicial (BC, BFS e SSSP); fração de vértices que são computadas por iteração; e se o processamento é focado no percorrimento do grafo (BFS, CC, SSSP e TC) ou no cálculo de propriedades dos vértices (BC e PR). Além disso, diferentes algoritmos têm diferentes aplicabilidades (e.g. SSSP em GPS's para fins de traçamento de caminhos, CC em recomendação de conexões em redes sociais como Twitter e Facebook, etc).

\section{MultGraph}

MultGraph é uma ferramenta offline que otimiza a execução simultânea de quaisquer conjunto de aplicações de grafos dado como entrada, encontrando os números de thre$a d s$ e ordem de execução ideais que melhorem o tempo de execução das aplicações e a eficiência do sistema. O fluxo de otimização o MultGraph é apresentado na Figura 1 e ele trabalha da seguinte forma: (i-Entradas) $\mathrm{O}$ usuário provê o conjunto de aplicações (algoritmos/grafos) assim como o sistema em que ele será executado; (ii-Heurística) $\mathrm{O}$ framework aplica uma heurística de dois passos onde: Passo 1, realiza uma categorização das aplicações com o objetivo de encontrar o melhor número de threads para cada uma delas baseando-se nos valores de eficiência. Também é feita a ordenação das aplicações de forma decrescente (do maior número de threads para o menor); e Passo 2, o MultGraph pega a lista de aplicações ordenadas e define a alocação das threads nos núcleos do sistema; (iii-Resultado) Por fim, o framework retorna um script com a solução obtida. Este script é usado para iniciar a execução paralela dos algoritmos/grafos dados em ( $i$ Entradas), cada um com seu número de threads devidamente configurado e agrupados na ordem dada como solução. A Figura 2 mostra um exemplo de solução gerada pelo MultGraph para o conjunto genérico de aplicações (TC-wikitalk, TC-youtube, BC-california, etc) em comparação com na maneira Tradicional de execução de aplicações em HPCs. Note que no MultGraph as aplicações são executadas simultaneamente, ao passo que na execução tradicional elas esperam em uma fila de até que as aplicações que chegaram antes terminem as suas execuções. 
O MultGraph considera no Passo 1 da (ii-Heurística) o seguinte cálculo de eficiência [Eager et al. 1989]: $E=\frac{t_{1}}{n \times t_{n}}$, onde $t_{1}$ é o tempo da execução serial, $t_{n}$ é o tempo da execução paralela usando $n$ threads. Considerando essa fórmula, o framework busca categorizar as aplicações de acordo com o número de threads que não implica em uma redução muito significativa na eficiência do sistema. Além disso, para o gerenciamento do número de threads e as suas alocações nos núcleos do sistema, o MultGraph usa as variáveis ambiente $O M P \_N U M_{-} T H R E A D S$ e GOMP_CPU_AFFINITY. Na seção seguinte, apresentamos os detalhes da heurística proposta.

\subsection{Heurística}

O Algoritmo 1 apresenta o pseudocódigo da heurística usada pela otimização do MultGraph. Ele toma como entradas o conjunto de aplicações a serem executadas $J$, o tamanho dos saltos no cálculo das eficiências slots e as configurações do multiprocessador alvo system. O algoritmo começa inicializando a variável que armazena as categorizações do Passo 1, opt_eff , com vazio ([]), o script da solução final também como vazio ( $\oslash)$ e a variável nucleos com o número de núcleos disponíveis no sistema (linhas 1-3).

Após isso, o algoritmo inicia o Passo 1 (linhas 5-15): Para cada aplicação $j$ na lista de aplicações a serem executadas $J$, o algoritmo coleta suas eficiências na execução com $N-i *$ slot threads, com $i$ variando no intervalo $\left[0, \frac{\mid \text { nucleos } \mid}{\text { slot }}-1\right]$ (e.g, considerando nucleos $=32$ e com slot $=8$ poderiam ser avaliadas as execução com 32, 24, 16, 8, 4 e 2 threads, nessa ordem). Dessa forma, a partir do maior número de threads aplicável ao número do tamanho do slot, testa-se a diferença de eficiência para com a de 2 threads e, caso essa seja menor que $20 \%$ (porcentagem alcançada por meio de uma análise experimental, que se mostrou ideal para uma boa variedade categórica), esse fica definido como o número otimizado de threads para essa aplicação (linha 9). No caso da diferença de eficiência nunca ser abaixo de $20 \%$, define-se a execução serial como sendo a melhor opção para a execução da aplicação (linha 13). No fim do Passo 1, a lista opt_eff é ordenada de forma decrescente (do maior valor para o menor) e inicia-se o Passo 2;

No Passo 2 (linha 17-25), o algoritmo define as execuções das aplicações por avaliar a lista opt_eff enquanto ainda existem aplicações a serem mapeadas nos núcleos do sistema (linha 17) e enquanto o sistema ainda tiver núcleos disponíveis (linha 18). Neste caso, o algoritmo obtém uma aplicação $j$ cujo seu número de threads com melhor eficiência $r$ (i.e., a tupla $<j, r>$ ) cabe no atual número de núcleos disponíveis no sistema |nucleos| (linhas 19). Após isso, define-se a afinidade de threads para os núcleos do sistema (linha 20) e escreve a solução no arquivo script (linha 21). No caso de não existirem núcleos disponíveis mesmo ainda tendo aplicações a serem executadas, uma nova rodada

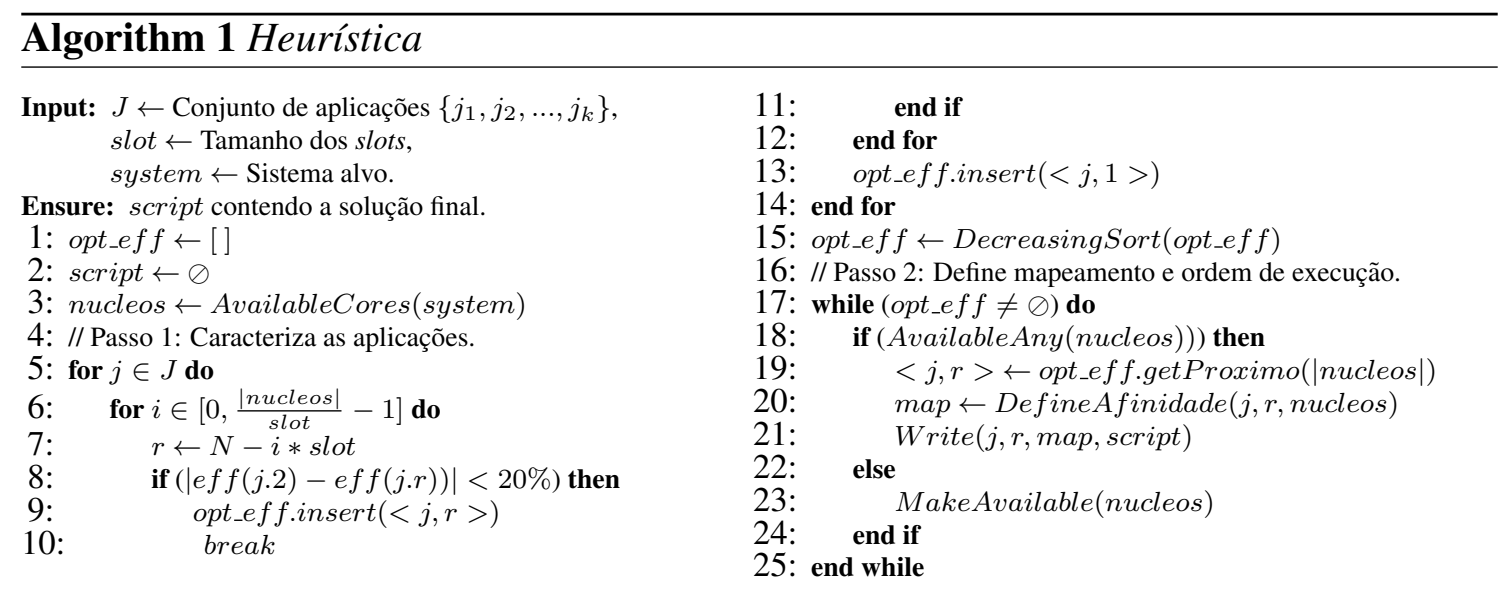


Tabela 4. Configurações das máquinas usadas.

\begin{tabular}{cccc}
\hline & Intel32 & Intel64 & AMD128 \\
\hline Processador & Intel Xeon E5-2640v2 & Intel Xeon X7550 & AMD Ryzen 3990X \\
Frequência base & $2,0 \mathrm{GHz}$ & $2,0 \mathrm{GHz}$ & $2,9 \mathrm{GHz}$ \\
\#Núcleos / \#Threads & $16 / 32$ & $32 / 64$ & $64 / 128$ \\
Cache L1 & $32 \mathrm{~KB}$ & $32 \mathrm{~KB}$ & $32 \mathrm{~KB}$ \\
Cache L2 & $256 \mathrm{~KB}$ & $256 \mathrm{~KB}$ & $512 \mathrm{~KB}$ \\
Cache L3 & $20 \mathrm{MB}$ & $18 \mathrm{MB}$ & $16 \mathrm{MB}$ \\
Memória RAM & $128 \mathrm{~GB}$ & $128 \mathrm{~GB}$ & $32 \mathrm{~GB}$ \\
\hline
\end{tabular}

do algoritmo inicia. Os núcleos são liberados (linha 23) e as aplicações são mapeadas nos núcleos para serem executadas assim que as aplicações previamente alocadas terminem suas execuções.

Complexidade do Tempo de Execução da Heurística: Para obter a complexidade assintótica do tempo de execução do algoritmo, devemos analisar cada um de seus passos: No Passo 1, temos que as linhas 5-12 tem complexidade $\mathrm{O}(|J| * \mid$ nucleos $\mid)$, assumindo o caso onde $s l o t=1$ (valor para a execução de pior caso). A ordenação realizada na linha 15 tem complexidade $\mathrm{O}(|J| * \log (|J|))$. Com isso, a complexidade do Passo 1 fica em $\mathrm{O}(|J| *[|n u c l e o s|+\log (|J|)])$. Para o Passo 2 temos uma complexidade de $\mathrm{O}(|J| * \mid$ nucleos $\mid)$, visto que dentro do loop que cobre as linhas 17-25, sempre 1 elemento é removido da lista opt_ef $f$ e todos os núcleos devem ser avaliados para saber se existe algum disponível. Portanto, a complexidade de pior caso do algoritmo é definida pelo Passo 1 e resulta em $\mathrm{O}(|J| *[|n u c l e o s|+\log (|J|)])$. Assim, considerando o custo polinomial na execução de pior caso, o MultGraph trabalha de forma eficiente, possibilitando ao usuário submeter batches de diferentes tamanhos a serem otimizados em sistemas com número variado de núcleos.

\section{Metodologia}

Algoritmos: Neste trabalho nós avaliamos os algoritmos disponíveis no GAP Benchmark Suite (GAPBS) [Beamer et al. 2015a]. O GAPBS disponibiliza a implementação de alto desempenho de um conjunto representativo de algoritmos de processamento de grafos, sendo um baseline representativo do estado da arte para as pesquisas nessa área. A ferramenta também conta com uma metodologia padronizada para a execução das aplicações, oferecendo confiabilidade nos dados extraídos em suas avaliações e comparações. Nós descrevemos todos os algoritmos avaliados na seção 2 (ver Tabela 3). Todos eles são implementados em C++11, paralelizados usando OpenMP e suas implementações consideram a estratégia de otimização que é mais adequada para cada um deles. Em nossos experimentos, nós compilamos as aplicações usando GNU g++ 10.1.0 e OpenMP versão 4.5 com a flag de otimização -O3.

Grafos: Nós avaliamos 10 grafos extraídos da SNAP [Leskovec and Krevl 2014]. Eles são descritos na seção 2 (ver Tabela 2). Nós consideramos um conjunto representativo de grafos do mundo real cobrindo as duas grandes classes de topologias (malhas e redes sociais) com diferentes características e tamanho (variando de 300 mil até 4 milhões de nós).

Ambiente de Execução: Nós realizamos os experimentos nos sistemas multicore apresentados na Tabela 4 usando o Sistema Operacional (SO) Linux com kernel v. 4.19.

Configurações e Análises Realizadas: Para analisar o comportamento dos algoritmos executando os grafos descritos anteriormente, nós consideramos tanto as métricas de processamento (e.g., tempo de execução e eficiência) quanto as que descrevem as características dos algoritmos e grafos (ver seção 2 para mais detalhes). Inicialmente, nós analisamos a escalabilidade das aplicações (ver seção 5.1), realizando execuções na versão serial e paralela, onde o número de threads varia de 2 ao máximo disponível no sistema 
(32, 64 e 128). Nessas execuções, o SO é responsável por decidir o mapeamento de threads aos núcleos. Todos os experimentos foram realizados com o número de execuções padrão especificado em [Beamer et al. 2015a]. Logo após, nós analisamos o desempenho do MultGraph (ver seção 5.2), mostrando as categorizações e os ganhos de desempenho e eficiência alcançados. Para essa análise, nós selecionamos aleatoriamente 4 batches (conjunto de algoritmos/grafos) com tamanhos variando de 3 a 39. Essa metodologia de criação dos batches destaca a capacidade do MultGraph em trabalhar com cenários reais, onde a otimização deve ser feita conforme as aplicações chegam, sem nenhum conhecimento a priori das mesmas. Os resultados do MultGraph são comparados com a execução padrão das aplicações em sistemas de HPC (baseline): as aplicações são executadas em sequencia, sempre usando o máximo de recursos de processamento disponíveis.

\section{Resultados Experimentais}

\subsection{Analise de escalabilidade}

Nesta seção, nós mostramos experimentalmente os dois princípios nos quais o MultGraph se baseia: (i) nem sempre usar o máximo de recursos do sistema entrega o melhor desempenho para as aplicações de grafos; e que (ii) não existe um número de threads ideal que resulta no melhor tempo de execução para todos os algoritmos e grafos. Nesse sentido, a Figura 3 apresenta um exemplo representativo do comportamento dos speedups sobre a versão serial para ( $a$ ) o algoritmo TC com relação a variação dos grafos e $(b)$ para o grafo cit-Patents com a variação dos algoritmos. Os experimentos foram executados no sistema AMD128. Desses dados, destacamos as seguintes observações: (i) O comportamento dos speedups na maioria dos algoritmos/grafos segue um padrão de crescimento até um ponto de saturação, onde não há mais ganhos significativos ou até a degradação do desempenho (e.g., TC-wik. satura com 94 threads com 44,10 de speedup, já quando executada com 128 threads tem speedup de 38,56); (ii) A magnitude dos ganhos em speedup varia conforme o algoritmo, grafo de entrada e sistema. Por exemplo, a execução TC-you. apresenta speedup de até 25,36, porém a TC-Cal. apresenta apenas 6,36. Já com relação a variação nos algoritmos, temos que o TC-cit. apresenta 13,66 e o SSSP-cit tem apenas 1,00 de speedup (i.e., não tem ganhos na execução paralela).

Tendo em vista a saturação dos speedups em pontos bem baixos do gráfico (32 threads para a maioria dos grafos em $(a)$, sendo que o TC é o algoritmo que mais provê escalabilidade), mostra que executar um conjunto de aplicações serialmente - uma após a outra com a total utilização do sistema - pode ser muito desvantajoso. Essa variação dos speedups mostrada na Figura 3 impacta diretamente no melhor número de threads para

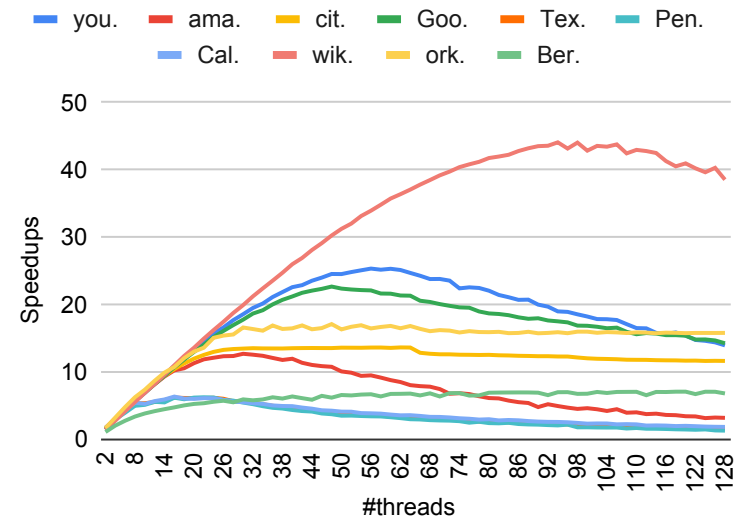

(a) $\mathrm{TC}$

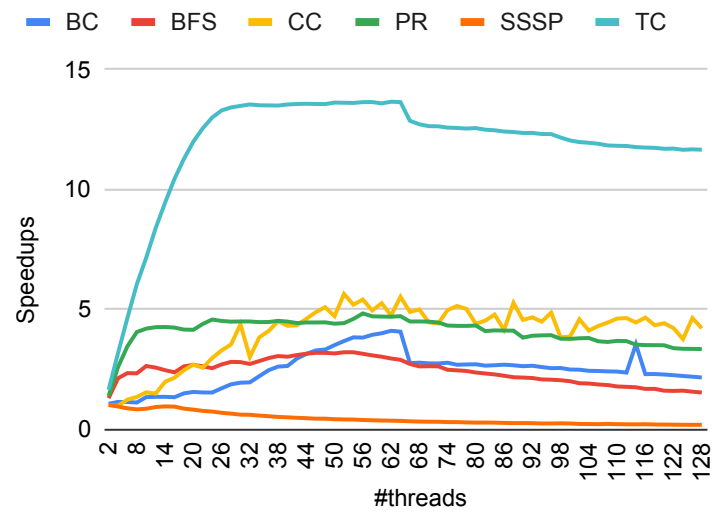

(b) cit-Patents

Figura 3. Speedups do algoritmo (a) TC e do grafo (b) cit-Patents quando executados na máquina AMD128. 
Tabela 5. \#threads com melhor tempo de execução para cada algoritmo/grafo.

\begin{tabular}{|c|c|c|c|c|c|c|c|c|c|c|c|c|c|c|c|c|c|c|}
\hline & \multicolumn{6}{|c|}{ Intel32 } & \multicolumn{6}{|c|}{ Intel64 } & \multicolumn{6}{|c|}{ AMD128 } \\
\hline & BC & BFS & $\mathrm{CC}$ & PR & SSSP & $\mathbf{T C}$ & BC & BFS & $\mathrm{CC}$ & PR & SSSP & TC & BC & BFS & $\mathrm{CC}$ & PR & SSSP & TC \\
\hline youtube & 28 & 16 & 26 & 28 & 4 & 32 & 64 & 62 & 64 & 64 & 14 & 62 & 16 & 16 & 62 & 8 & 6 & 56 \\
\hline ama & 10 & 6 & 28 & 18 & 4 & 22 & 64 & 62 & 64 & 64 & 62 & 62 & 8 & 14 & 18 & 18 & & 30 \\
\hline citPs & 14 & 14 & 32 & 32 & 6 & 32 & 64 & 62 & 62 & 64 & 62 & 62 & 62 & 52 & 52 & 56 & 2 & 62 \\
\hline Go & 32 & 30 & 28 & 30 & 14 & 30 & 64 & 64 & 62 & 62 & 12 & 64 & 16 & 16 & 32 & 12 & 8 & 48 \\
\hline Tex & 30 & 18 & 32 & 30 & 2 & 20 & 24 & 44 & 64 & 62 & 2 & 62 & 8 & 8 & 46 & 58 & 2 & 16 \\
\hline Pen & 26 & 18 & 32 & 30 & 2 & 16 & 28 & 64 & 62 & 62 & 2 & 62 & 8 & 8 & 40 & 58 & 2 & 22 \\
\hline Cali & 30 & 30 & 30 & 30 & 2 & 26 & 38 & 58 & 62 & 64 & 2 & 64 & 8 & 8 & 50 & 112 & 2 & 16 \\
\hline wikitalk & 30 & 24 & 14 & 26 & 22 & 30 & 62 & 64 & 62 & 62 & 34 & 58 & 16 & 14 & 12 & 10 & 8 & 94 \\
\hline orkı & 32 & 30 & 30 & 32 & 22 & 32 & 62 & 64 & 64 & 64 & 20 & 64 & 24 & 16 & 66 & 12 & 16 & 48 \\
\hline Berk & 18 & 14 & 24 & 32 & 2 & 32 & 8 & 62 & 62 & 64 & 4 & 62 & 8 & 8 & 66 & 34 & 6 & 120 \\
\hline
\end{tabular}

executar cada combinação algoritmo/grafo. Na Tabela 5 nós sumarizamos o número de threads que apresentou o melhor tempo de execução para cada algoritmo/grafo nos três sistemas avaliados (Intel32, Intel64 e AMD128). Alguns algoritmos tendem a ter melhor performance com um maior número de threads (CC e TC em todos os sistemas), porém também existem aqueles que requerem apenas de um número pequeno de threads (SSSP). Vários aspectos influenciam esse comportamento: os diferentes algoritmos realizam diferentes tipos de computação (ver Tabela 3); os grafos do mundo real possuem diferentes topologias (ver Tabela 2); e, os diversos multiprocessadores apresentam diferente número de núcleos e hierarquia de memória (ver seção 4). Todos esses aspectos devem ser considerados quando se ajusta o número de threads para melhorar a execução dos algoritmos de grafos, pois uma otimização específica para um algoritmo, grafo, ou sistema, não será necessariamente efetiva se qualquer uma dessas variáveis mudar. Com isso, a vantagem do MultGraph é a capacidade de contornar os aspectos acima mencionados, otimizando a execução simultânea das aplicações de grafos com base na categorização das aplicações.

\subsection{Performance do MultGraph}

\subsubsection{Passo 1: Categorização dos algoritmos/grafos:}

Nessa seção nós apresentamos o resultado da aplicação do Passo 1 da Heurística do MultGraph (ver seção 3). Esse passo é responsável por categorizar as aplicações com relação ao número de threads que entrega a melhor eficiência do sistema. Na Tabela 6, nós detalhamos o melhor número de threads para cada sistema avaliado (Intel32, Intel64 e AMD128).

No geral, a maior parte das aplicações tem sua melhor execução com 1 e 8 threads. Mais especificamente, para o Intel32, 51,7\% das aplicações são categorizadas com 1 thread e 58,3\% e 60\%, respectivamente, para o Intel64 e o AMD128. Nessa mesma ordem, as aplicações de 8 threads aparecem com uma porcentagem de 16,7\%, 41,7\% e $25 \%$. Isso acontece principalmente nos algoritmos BC e SSSP em todas as máquinas e para os demais algoritmos (com exceção do TC nas máquinas com maior número de núcleos). Um maior número de threads implica em maior utilização dos resursos do sistema e maior overhead de sincronização nos acessos aos dados compartilhados. Se os recursos utilizados ficarem muito tempo ociosos quando na sincronização dos dados, a eficiência do sistema é prejudicada, fazendo com que a redução do número de threads apresente melhor resultado.

\subsection{Passo 2: Execução multiprogramada}

Esta seção discute os resultados da execução dos scripts gerados na aplicação do Passo 2 do MultGraph. Esse passo é responsável por definir a alocação das aplicações nos núcleos do sistema assim como sua ordem de execução. Na Figura 4 nós apresentamos o tempo de execução nos 4 diferentes batches/conjuntos de aplicações (grupos de barras). Os resultados são apresentados para os diferentes sistemas e normalizados pelo baseline 
Tabela 6. \#threads com melhor eficiência para cada algoritmo/grafo.

\begin{tabular}{|c|c|c|c|c|c|c|c|c|c|c|c|c|c|c|c|c|c|c|}
\hline & \multicolumn{6}{|c|}{ Intel32 } & \multicolumn{6}{|c|}{ Intel64 } & \multicolumn{6}{|c|}{ AMD128 } \\
\hline & BC & BFS & $\mathrm{CC}$ & PR & SSSP & TC & BC & BFS & $\mathrm{CC}$ & PR & SSSP & TC & BC & BFS & $\mathrm{CC}$ & PR & SSSP & TC \\
\hline youtube & 1 & 8 & 1 & 8 & 1 & 16 & 8 & 8 & 8 & 1 & 1 & 8 & 1 & 8 & 1 & 1 & 1 & 40 \\
\hline amazon & 8 & 24 & 1 & 24 & 1 & 32 & 8 & 8 & 1 & 1 & 1 & 8 & 1 & 8 & 8 & 1 & 1 & 16 \\
\hline citPatents & 8 & 8 & 1 & 24 & 1 & 32 & 8 & 8 & 1 & 8 & 8 & 1 & 1 & 1 & 1 & 8 & 1 & 16 \\
\hline Google & 1 & 16 & 1 & 32 & 1 & 32 & 1 & 8 & 1 & 8 & 1 & 8 & 8 & 8 & 1 & 8 & 1 & 40 \\
\hline Texas & 1 & 1 & 32 & 1 & 1 & 16 & 1 & 1 & 8 & 8 & 1 & 1 & 1 & 1 & 32 & 1 & 1 & 8 \\
\hline Pennsylvania & 1 & 1 & 32 & 1 & 1 & 24 & 1 & 8 & 8 & 1 & 1 & 1 & 1 & 1 & 32 & 1 & 1 & 1 \\
\hline California & 1 & 1 & 32 & 1 & 1 & 16 & 1 & 1 & 1 & 1 & 1 & 1 & 8 & 1 & 1 & 1 & 1 & 8 \\
\hline wikitalk & 1 & 16 & 1 & 1 & 8 & 32 & 1 & 8 & 1 & 1 & 1 & 8 & 1 & 1 & 1 & 1 & 1 & 80 \\
\hline orkut & 1 & 8 & 1 & 32 & 1 & 24 & 1 & 8 & 1 & 1 & 1 & 8 & 8 & 8 & 1 & 16 & 8 & 16 \\
\hline Berk & 1 & 1 & 8 & 8 & 1 & 8 & 1 & 1 & 8 & 8 & 1 & 8 & 1 & 1 & 8 & 1 & 1 & 8 \\
\hline
\end{tabular}

(as execuções das aplicações em sequência sempre usando o máximo número de threads), logo quanto maior as barras, melhor é o desempenho do MultGraph.

Como o MultGraph baseia seu processo de otimização no cálculo da eficiência, o melhor número de threads encontrado para cada aplicação vai ser reduzido na maioria dos casos (ver Tabela 6). Isso beneficia a execução de vários algoritmos de grafos simultaneamente, o que implica em melhora na eficiência do sistema e no tempo de execução total das aplicações. No geral, considerando todos os conjuntos de aplicações avaliados, o MultGraph apresenta ganhos de 1,91× no Intel32, 9, $21 \times$ no Intel64 e 2, 45× no AMD128 para os tempos de execução. Considerando cada conjunto de aplicações individualmente, o MultGraph melhora em até $3,06 \times, 16,53 \times, 2,10 \times$ e 29, $48 \times$ o tempo de execução dos quatro respectivos conjuntos.

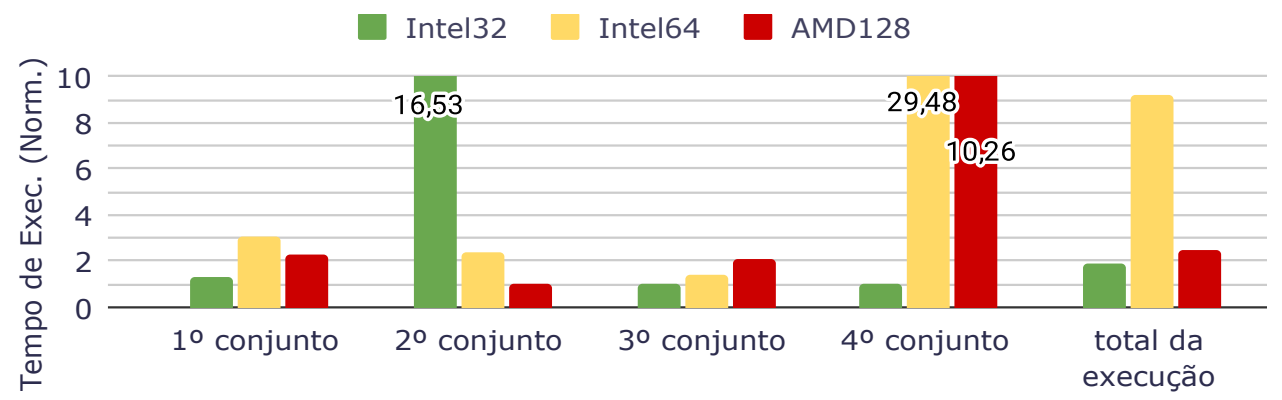

Figura 4. Resultados de tempo de execução nas soluções obtidas pelo MultGraph com relação ao baseline (valores acima de 1 indicam em quantas vezes o MultGraph é melhor).

\section{Trabalhos Relacionados}

Nessa seção, apresentamos trabalhos da literatura que focam sua otimização na: (i) execução multiprogramada de aplicações em sistemas multiprocessados; (ii) análise e otimização de algoritmos de grafos.

(i) Georgios Varisteas [Varisteas 2015] propõe uma solução com o objetivo de otimizar da taxa de transferência das aplicações que estão executando concorrentemente. Sudarsan e Ribbens [Sudarsan and Ribbens 2016] apresentam um framework que torna dinâmico o dimensionamento das aplicações. Este dimensionamento é baseado em cenários e estratégias onde prioriza-se o menor tempo de execução utilizando todos os recursos disponíveis e em uma outra situação prioriza-se as execuções de alta ou baixa prioridade utilizando os recursos dentro de um limite pré-determinado. Jorge González-Domínguez [Jorge González-Domínguez and Touriño 2012] desenvolve um algoritmo que usa o Servet benchmark suite para o mapeamento de aplicações paralelas em sistemas multicore e que analisa o seu impacto por meio de três modelos de programação paralela diferentes: troca de mensagens, memória compartilhada e espaço de memória global particionado. O trabalho de [da Silva et al. 2021] propõe o SRA (Smart Resource 
Allocation), um algoritmo que otimiza tempo de execução das aplicações e o consumo de energia do sistema por executar simultaneamente várias aplicações, cada uma com os seus melhores número de threads.

(ii) O trabalho de [Shun and Blelloch 2013] propõe Ligra, um modelo de programação de memória compartilhada para algoritmos de grafos que ajusta automaticamente a maneira como o grafo é avaliado de acordo com um limiar provido pelo programador. O trabalho de [Zhang et al. 2015] realiza um extenso estudo sobre os impactos da execução de algoritmos de grafos em sistemas NUMA. Com base nisso, eles propõe Polymer, um framework que otimiza o processamento baseando-se na localidade dos dados e em sua distribuição nas memórias do sistema. Amitabha Roy [Roy et al. 2013] propõe $\mathrm{X}$-Stream, um framework para processamento de grafos que utiliza o modelo scattergather centrado em arestas. Por ser capaz de evitar acessos aleatórios à memória principal, X-Stream apresenta boa escalabilidade conforme o aumento no número de núcleos do sistema.

Contribuição: Por mais que diversos trabalhos tenham proposto a otimização da performance de aplicações paralelas, ao avaliar a sua execução simultânea em processadores multicore $(i)$, com base nos trabalhos avaliados, nós somos os primeiros a considerar a otimização de algoritmos de grafos, tendo em vista tanto o tempo de execução do algoritmo quanto a eficiência do sistema. Além do mais, nossa proposta ortogonaliza os esforços de (ii), uma vez que o MultGraph melhora a performance dos algoritmos de processamento de grafos sem requerer qualquer mudança em seus códigos fonte (i.e., o algoritmo BFS nas diferentes implementações do Ligra, X-Stream ou Polymer pode ter melhor tempo de execução em utilizar o nosso framework).

\section{Conclusão}

Neste trabalho nós mostramos que limitações de escalabilidade dos algoritmos de processamento de grafos variam de acordo com as suas características e as topologias dos grafos processados. Com base nisso, nós propomos o MultGraph: uma estratégia de multiprogramação que, dado conjunto de aplicações e o multiprocessador alvo, encontra a melhor configuração de algoritmo/grafo que deve ser executada simultaneamente de forma a melhorar o tempo de execução e a eficiência do sistema. Os resultados experimentais considerando três diferentes processadores multicores (Intel e AMD) mostraram que o MultGraph melhora em até 9, $21 \times$ o tempo de execução dos algoritmos em relação à execução padrão de aplicações em sistemas HPC. Nossas perspectivas de trabalhos futuros incluem uma analise mais detalhada do comportamento de execução das aplicações com base em dados de baixo nível (de processamento e acessos à memória) e em uma análise do comportamento do MultGraph quando na otimização de diferentes frameworks de processamento de grafos.

\section{Agradecimentos}

Esse estudo foi financiado em parte pela Coordenação de Aperfeiçoamento de Pessoal de Nível Superior - Brasil (CAPES) - Finance Code 001, a Fundação de Amparo à Pesquisa do Estado do RS (FAPERGS) e o Conselho Nacional de Desenvolvimento Científico e Tecnológico $(\mathrm{CNPq})$. Alguns experimentos desse trabalho usaram a infraestrutura do PCAD, http://gppd-hpc.inf.ufrgs.br/, do INF/UFRGS.

\section{Referências}

Beamer, S., Asanović, K., and Patterson, D. (2015a). The gap benchmark suite. arXiv preprint arXiv:1508.03619.

Beamer, S., Asanovic, K., and Patterson, D. (2015b). Locality exists in graph processing: Workload characterization on an ivy bridge server. In $I S W C$, pages 56-65. IEEE. 
da Silva, V., Medeiros, T., Rocha, H., Luizelli, M., Rossi, F., Beck, A. C., and Lorenzon, A. (2020). Análise da execução concorrente de aplicações paralelas em arquiteturas multicore. In WSCAD, pages 61-72. SBC.

da Silva, V. S., Nogueira, A. G., de Lima, E. C., de A. Rocha, H. M., Serpa, M. S., Luizelli, M. C., Rossi, F. D., Navaux, P. O., Beck, A. C. S., and Francisco Lorenzon, A. (2021). Smart resource allocation of concurrent execution of parallel applications. $C C P E$, page e6600.

Eager, D., Zahorjan, J., and Lazowska, E. (1989). Speedup versus efficiency in parallel systems. IEEE TC, 38(3):408-423.

Gleich, D. F. (2014). Pagerank beyond the web.

Heidari, S., Simmhan, Y., Calheiros, R., and Buyya, R. (2018). Scalable graph processing frameworks: A taxonomy and open challenges. CSUR, 51:1-53.

Jorge González-Domínguez, Guillermo L. Taboada, B. B. F. M. J. M. and Touriño, J. (2012). Automatic mapping of parallel applications on multicore architectures using the servet benchmark suite. CEE, 38:258-269.

Leskovec, J. and Krevl, A. (2014). SNAP Datasets: Stanford large network dataset collection. http://snap. stanford.edu/data.

Lorenzon, A. F. and Beck Filho, A. C. S. (2019). Parallel computing hits the power wall: principles, challenges, and a survey of solutions. Springer Nature.

Mofrad, M. H., Melhem, R., Ahmad, Y., and Hammoud, M. (2020). Graphite: a numaaware hpc system for graph analytics based on a new mpi* $\mathrm{x}$ parallelism model. Proceedings of the VLDB Endowment, 13(6):783-797.

Raasch, S. E. and Reinhardt, S. K. (2003). The impact of resource partitioning on smt processors. In PACT, pages 15-25.

Rocha, H. M. G. d. A., Schwarzrock, J., Lorenzon, A. F., and Beck, A. C. S. (2021). Boosting graph analytics by tuning threads and data affinity on numa systems. In $P D P$, pages 161-168. IEEE.

Roy, A., Mihailovic, I., and Zwaenepoel, W. (2013). X-stream: Edge-centric graph processing using streaming partitions. page 472-488.

Shun, J. and Blelloch, G. E. (2013). Ligra: a lightweight graph processing framework for shared memory. In ACM PPoPP, pages 135-146.

Sudarsan, R. and Ribbens, C. J. (2016). Combining performance and priority for scheduling resizable parallel applications. JPDC, 87:55-66.

Suleman, M. A., Qureshi, M. K., and Patt, Y. N. (2008). Feedback-driven threading: Power-efficient and high-performance execution of multi-threaded workloads on cmps. SIGARCH CAN, 36(1):277-286.

Varisteas, G. (2015). Effective cooperative scheduling of task-parallel applications on multiprogrammed parallel architectures. PhD thesis. QC 20151016.

Yan, D., Bu, Y., Tian, Y., and Deshpande, A. (2017). Big graph analytics platforms. FTD, 7(1-2):1-195.

Zhang, K., Chen, R., and Chen, H. (2015). Numa-aware graph-structured analytics. SIGPLAN Not., 50(8):183-193. 